\section{Building Huntington's monkeys}

\section{By Brian Moy, Staff Writer}

Efforts to develop treatments for Huntington's disease have been hampered by the lack of utility of rodent models, which do not satisfactorily replicate the physiological and behavioral changes seen in disease patients. ${ }^{1}$ In a paper published in Nature, researchers at Yerkes National Primate Research Center at Emory University describe the development of a transgenic rhesus monkey that at least initially presents with clinical features of the human disease.

Company representatives and academics contacted by SciBX agree the new model could pave the way for developing Huntington's disease (HD) therapies if it is possible to breed the monkeys and develop lines with consistent genotypes and phenotypes.

$\mathrm{HD}$ is an autosomal dominant neurodegenerative disorder characterized by motor impairment, cognitive deterioration and psychiatric disturbances. The disease is caused by expansion of cytosine-adenine-guanine (CAG) trinucleotide repeats in the first exon of the human huntingtin (HTT) gene.

In the Nature paper, the researchers injected lentiviruses expressing exon one of human HTT with 84 CAG repeats and lentiviruses expressing the GFP gene into unfertilized mature rhesus oocytes.

Following intracytoplasmic sperm injection and embryo transfer to eight surrogates, six pregnancies were established and five live newborns were delivered at full term. All of the monkeys carried the transgenic mutant HTT and GFP.

Three of the newborns carried between two to four copies of mutant HTT. Of these, two had respiratory difficulties, displayed signs of motor impairment and survived for less than a day. The third developed dystonia and chorea two days after birth and died a month later.

The two surviving monkeys, which only carried a single copy of mutant HTT, have 29 and 83 CAG repeats, respectively. The monkey with 29 CAG repeats, a value within the normal range for humans without HD, appears to be normal and healthy, displaying no disease symptoms. The monkey with 83 CAG repeats displayed mild involuntary motor movement typical of HD that appeared sporadically within one week of birth. Similar features were seen in the three monkeys that did not survive.

"Our HD monkeys develop the clinical features of human HD patients that no other animal has been able to present, including dys- tonia, chorea and bradykinesia," said Anthony Chan, principal investigator and corresponding author of the Nature paper. He is a research assistant professor in the Division of Neuroscience at Yerkes and an assistant professor in the Department of Human Genetics at Emory University School of Medicine.

"An animal model that can recapitulate human disease will allow us to more precisely determine the efficacy of potential therapeutics, as they should have comparable if not identical impact in human patients," Chan said.

The researchers are continuing to monitor for disease progression in the surviving monkeys using MRI, cognitive behavioral tests and blood tests. Peripheral blood samples are being collected for genomic, proteomic and metabolite profiling.

They are monitoring both monkeys because the researchers do not know the susceptibility of HD in the monkey with the shorter repeat that has not developed any symptoms.

\section{Model behavior}

The transgenic monkey could be useful for investigating the mechanism underlying HD progression as well as for screening potential therapeutics.

Steven Finkbeiner, an associate director and associate investigator at the Gladstone Institute of Neurological Disease and an associate professor of neurology and physiology at the University of California, San Francisco (UCSF), told SciBX that "the biggest advantage of the model is that a human's neuronal circuits are more closely related to monkeys than they are to mice. The similarities could allow for more detailed studies into the role of neuronal dysfunction in HD patients."

"Although the paper is lacking detailed descriptions of the clinical phenotype of the monkeys," Joakim Tedroff, medical director at NeuroSearch A/S, noted that "it does reveal some very interesting pathological findings, such as nuclear inclusions and neuropil aggregates that were observed in the brains of the monkeys."

Nuclear inclusions, which are aberrant accumulations of mutant HTT in the nucleus of brain cells, and neuropil aggregates, which are cytoplasmic aggregates of mutant HTT localized in the neuronal processes, including axons and dendrites, are hallmark neuropathological features of HD.

NeuroSearch's ACR16, a dopamine stabilizer, is in Phase III testing to treat HD.

"The monkeys, which appear to have the genotype and phenotype of HD patients, could be very useful for validating drugs for HD," said Belinda Tsao Nivaggioli, chairman and CEO of Avicena Group Inc. "For many years we have relied on rodent models to screen for HD drugs. Unfortunately, there are a lot of failures when going from a mouse model to humans." 
Avicena is developing HD-02, which it describes as a pharmaceutical-grade ultrapure creatine monohydrate. The company plans to begin a Phase III trial to treat early-stage HD patients by year end.

Nancy Wexler, professor of neuropsychology at Columbia University Medical School and president of the Hereditary Disease Foundation, suggested the monkey model will be useful for preclinical testing of gene delivery and silencing technologies for HD. The foundation supports research aimed at developing RNAi and antisense therapies for treating HD.

"We need to determine if using RNAi gene therapy to shut off, or even reduce, production of the huntingtin gene-both the normal and abnormal forms of the protein-can safely prevent Huntington's disease," she told SciBX. "The nonhuman primate model is critical for answering this essential question."

\section{Monkey problems}

Before the model is ready for prime time, several issues need to be addressed, including determining how the disease progresses in the surviving monkeys as they age. Another question is whether the monkeys will be able to reproduce and, if so, whether successive generations will have consistent genotypes and phenotypes.

Chan said it is too early to determine whether germline integration has even occurred.

"As the monkeys grow, it will be important to see if they behave like an HD patient," said Avicena's Nivaggioli. “There should be certain deteriorations as they age, including movement, psychological and orientation disorders."

NeuroSearch's Tedroff agreed. "In order to use the model for screening potential drugs, further research needs to investigate the behavioral and cognitive phenotypes that develop in the monkeys and compare them to symptoms that develop in HD patients."

Some researchers believe that modeling HD in a genetically precise way using the entire HTT gene is important to accurately study the disease. The Yerkes study used lentiviruses expressing only exon one of human HTT.

"Ideally, in order to fully capture the HD phenotype in the model, you need a vector with a full-length, physiologically relevant HD transgene," said David Rubinsztein, professor of molecular neurogenetics at the University of Cambridge. "If the monkeys have the appropriate transgene, we would be able to screen certain compounds for their ability to delay the onset of disease."

UCSF's Finkbeiner disagreed. "While there probably are some differences in phenotype by using a full-length huntingtin gene compared to a fragment of the gene, it seems that the majority of the fundamental mechanisms behind HD are still in play when using a fragment," he said.

However, Finkbeiner said, "one problem is that it is unclear whether the monkeys would be able to breed. If they can breed, it would be interesting to see if the lentivirus is propagated through the germline."

If the monkeys can't breed, he said, "it becomes difficult to establish a consistent model and compare results from one monkey to another."

\section{REFERENCES}

1. Yang, S.-H. et al. Nature; published online May 18, 2008;

doi:10.1038/nature06975

Contact: Anthony Chan, Yerkes National Primate Research Center, Atlanta, Ga.

e-mail: achan@genetics.emory.edu

\section{COMPANIES AND INSTITUTIONS MENTIONED}

Avicena Group Inc. (OTCBB:AVGO), Palo Alto, Calif.

Columbia University Medical School, New York, N.Y.

Emory University, Atlanta, Ga.

Gladstone Institute of Neurological Disease, San Francisco, Calif.

Hereditary Disease Foundation, New York, N.Y.

NeuroSearch A/S (CSE:NEUR), Ballerup, Denmark

University of California, San Francisco, Calif.

University of Cambridge, Cambridge, U.K. 